metro-hs-ph1

AP-SU-00/02

hep-ph/0012166

Dec, 2000

\title{
Single scalar top production with polarized beams in $e p$ collisions at HERA
}

\author{
Shoichi Kitamura \\ Tokyo Metropolitan University of Health Sciences, Tokyo 116-8551, Japan \\ kitamura@post.metro-hs.ac.jp \\ Tadashi Kon \\ Faculty of Engineering, Seikei University, Tokyo 180-8633, Japan \\ kon@apm.seikei.ac.jp \\ Tetsuro Kobayashi \\ Faculty of Engineering, Fukui Institute of Technology, Fukui 910-8505, Japan \\ koba@ge.seikei.ac.jp
}

\begin{abstract}
From the point of view of the R-parity breaking supersymmetric model, we propose a scalar top (stop) search with longitudinally polarized electron $\left(e_{L}^{-}\right)$ and positron $\left(e_{R}^{+}\right)$beams which will soon be available at the upgraded HERA. Fully polarized $e^{-}$or $e^{+}$beams could produce the stop two times as much as unpolarized beams, while they increase background events due to the process of the standard model by $\sim 30 \%$ in comparison with unpolarized ones. We show that right-handed $e^{+}$beams at HERA is efficient to produce the stop in the model. With $1 \mathrm{fb}^{-1}$ of integrated luminosity we estimate reach in the coupling constant $\lambda_{131}^{\prime}$ for masses of the stop in the range 160-400 GeV. We can set a $95 \%$ confidence-level exclusion limit for $\lambda_{131}^{\prime}>0.01-0.05$ in the stop mass range of $240-280 \mathrm{GeV}$ if no singal of the stop is observed. We also point out that $y\left(=Q^{2} / s x\right)$ distributions of $e^{+}$coming from the stop shows the different behavior from those of the standard model.
\end{abstract}




\section{Introduction}

In supersymmetric (SUSY) extension of the standard model(SM), a bosonic superpartner (sfermion) is assigned to every fermionic SM particle and vice versa [1, 2]. Helicity states of the SM quark $q_{L}$ and $q_{R}$ acquire SUSY partners $\widetilde{q}_{L}$ and $\widetilde{q}_{R}$, which are also the mass eigenstates for the first two generations to a good approximation. On the other hand, a large mixing is expected between the left- and right-handed states of scalar SUSY partner in the third generation because of the large mass of the fermionic SM particles [3]. This leads to a possible large splitting between the mass states, which implies the existence of a lighter scalar top quark(stop, $\widetilde{t_{1}}$ ) lighter than the top quark and the other squarks.

H1 and ZEUS reported an event excess relative to the SM expectation at the large Bjorken parameter $x$ and high four-momentum transfer squared $Q^{2}$ in the deep inelastic scattering (DIS), $e^{+} p \rightarrow e^{+} X$ 国, 河. Although the excess became less significant recently with increase of statistics, this fact implies that HERA is a powerful machine to open the door beyond the SM as leptoquark models and SUSY models with R-parity breaking (RB) interactions [6].

In this letter, we estimate production cross sections of the s-channel resonance of a single stop through RB interactions, $e p \rightarrow \widetilde{t_{1}} X \rightarrow e X$ with longitudinally polarized $e^{-}$ and $e^{+}$beams in the framework of the Minimal SUSY Standard Model(MSSM). Single stop production $e q \rightarrow \widetilde{t}_{1} \rightarrow e q$ in the neutral current processes gives the sharp peak in the $x$ distribution of the scattered $e^{-}$and $e^{+}$, and this peak is enhanced in the high $Q^{2}$ region. We show that right handed $e^{+}$beams are more efficient to produce the stop than unpolarized ones or $e^{-}$beams in the model. HERA will soon be upgraded, and experiments using polarized $e^{-}$or $e^{+}$beams with high luminosity will start[0]. Then we are able to search for the stop with high statistics in the wide mass range even for smaller values of the coupling of the RB interactions than before.

\section{$2 \quad$ Theoretical models}

We are based on the minimal MSSM with RB interactions

$$
L=\lambda_{1 j k}^{\prime}\left(\widetilde{u_{j L}} \overline{d_{k}} P_{L} e-\overline{\overline{d_{k R}}} \overline{e^{c}} P_{L} u_{j}\right)+h . c
$$

where $i, j, k$ are generation indices and $P_{L, R}$ are left and right handed chiral projection operators, respectively. The Lagrangian Eq. (11) will be most suitable for the ep collider experiments at HERA because the squarks will be produced in the $s$-channel in $e^{ \pm}-q$ sub-processes.

$$
\begin{aligned}
e^{+}\left(e^{-}\right)+d_{k}\left(\overline{d_{k}}\right) & \rightarrow \widetilde{u_{j L}}\left(\overline{\overline{u_{j L}}}\right) \rightarrow e^{+}\left(e^{-}\right)+d_{k}\left(\overline{d_{k}}\right) \\
e^{+}\left(e^{-}\right)+\overline{u_{j}}\left(u_{j}\right) & \rightarrow \widetilde{\widetilde{d_{k R}}}\left(\widetilde{d_{k R}}\right) \rightarrow e^{+}\left(e^{-}\right)+\overline{u_{j}}\left(u_{j}\right) .
\end{aligned}
$$

Note that the squark $\widetilde{u_{j L}}$ or $\widetilde{d_{k R}}$ cannot couple to any neutrinos via the $R$-breaking interactions. This is a unique property of the squark scenarios which could be useful for us to distinguish the squarks from some leptoquarks. 
Here we pay attention to the stop production via $\lambda_{131}^{\prime} \neq 0 \mathrm{RB}$ interaction because it could be the lightest squark in the MSSM. The stops

$\left(\widetilde{t_{L}}, \widetilde{t_{R}}\right)$ are naturally mixed each other [3, 8] due to a large top quark mass [9] and the mass eigenstates $\left(\widetilde{t}_{1}, \widetilde{t}_{2}\right)$ are parametrized by a mixing angle $\theta_{t}$,

$$
\begin{aligned}
& \widetilde{t}_{1}=\widetilde{t_{L}} \cos \theta_{t}-\widetilde{t_{R}} \sin \theta_{t}, \\
& \tilde{t}_{2}=\widetilde{t_{L}} \sin \theta_{t}+\widetilde{t_{R}} \cos \theta_{t} .
\end{aligned}
$$

In this case the interaction Lagrangian (1) for the lighter stops $\widetilde{t}_{1}$ is written by

$$
L=\lambda_{131}^{\prime} \cos \theta_{t}\left(\widetilde{t_{1}} \bar{d} P_{L} e+\widetilde{t}_{1}^{*} \bar{e} P_{R} d\right),
$$

which generates the s-channel stop $\widetilde{t}_{1}$ production in the neutral current(NC) process 10]:

$$
e^{ \pm} p \rightarrow \widetilde{t_{1}} X \rightarrow e^{ \pm} q X
$$

and the relevant Feynman diagrams are depicted in Fig.1. In the calculation, the contributions of the s-channel and the u-channel exchange processes of the stop are estimated separately, where interferences with amplitudes of the SM shown in Fig.1 are taken into account. We calculate the inclusive differential cross section for the NC processes $e^{ \pm} p \rightarrow e^{ \pm} q X$ with longitudinally polarized $e_{L, R}^{ \pm}$beams:

$$
\frac{d \sigma}{d x d Q^{2}}\left[e_{L, R}^{ \pm}\right]=\frac{2 \pi \alpha^{2}}{x^{2} s^{2}} \sum_{q}\left[q\left(x, Q^{2}\right) \sum_{i} T_{i}\left(e_{L, R}^{ \pm} q\right)+\bar{q}\left(x, Q^{2}\right) \sum_{i} T_{i}\left(e_{L, R}^{ \pm} \bar{q}\right)\right]
$$

where $q\left(x, Q^{2}\right)$ 's are the quark distribution functions in the proton. The index $i$ represents each diagram in Fig.1 and their interferences. The analytic expression for the coefficients $T_{i}\left(e_{L, R}^{ \pm} q\right)$ is explicitly given in [11].

The cross section depends sensitively on the decay width of the stop. In this calculation, we assume $\operatorname{BR}\left(\widetilde{t_{1}} \rightarrow e d\right) \simeq 100 \%$, i.e.,

$$
\Gamma_{\widetilde{t_{1}}}=\frac{\alpha}{4} F_{R B}\left(\widetilde{t_{1}}\right) m_{\widetilde{t_{1}}},
$$

where the coupling strength $F_{R B}\left(\widetilde{t_{1}}\right)$ is defined as

$$
F_{R B}\left(\widetilde{t_{1}}\right)=\frac{\lambda_{131}^{\prime} \cos ^{2} \theta_{t}}{4 \pi \alpha}
$$

We expect a clear signal of the stop as a sharp peak in the Bjorken parameter $x$ distribution at the position of $x=m_{\widetilde{t}_{1}}^{2} / s$. We point out from(6) that the left-handed polarized electron $\left(e_{L}^{-}\right)$and the right-handed polarized positron $\left(e_{R}^{+}\right)$beams could be advantageous to produce the stop. Furthermore the $e^{+}$beam is more efficient than the $e^{-}$one to separate the stop signal from the SM background, which can be easily understood from the structure of the coupling (6). While the $e^{-}$collides only with sea $\bar{d}$-quarks in the proton , the $e^{+}$collides with valence $d$-quarks to produce the stop. From the difference of the structure function of the proton we can expect $\sigma\left(e^{+} d \rightarrow \widetilde{t_{1}}\right)>\sigma\left(e^{-} \bar{d} \rightarrow \overline{\widetilde{t_{1}}}\right)$. Throughout the present work, we use the CTEQ4M for the parton distribution function[12]. 


\section{Cross sections of the stop production}

We have calculated the differential cross sections eq.(8) using the program package BASES [13], where the energies $E_{e}=30 \mathrm{GeV}$ and $E_{p}=820 \mathrm{GeV}(\sqrt{s}=314 \mathrm{GeV})$ are assumed. Event generation of $e^{ \pm} p \rightarrow e^{ \pm} q X$ has been carried out by using SPRING 13] following the numerical results of cross sections obtained by BASES. In the previous work, we found that the lower $Q^{2}$ cuts are efficient to supress the SM background since the s-channel stop contribution is independent of $Q^{2}$ [11]. In what follows we impose the condition $Q^{2}>10^{4}$ $(\mathrm{GeV} / \mathrm{c})^{2}$ under $Q_{\max }^{2}=98000(\mathrm{GeV} / \mathrm{c})^{2}$.

Fig. 2 shows cross sections of the stop production together with those of the SM against the degree of polarization of $e^{-}$and $e^{+}$beams. Suggested by H1 and ZEUS reports [ [1, 5], we assume here $m_{\widetilde{t}_{1}}=200 \mathrm{GeV}$ and $\lambda_{131}^{\prime}=0.01,0.02,0.03$. These values of $\lambda_{131}^{\prime}$ are those below the present upper bound for this value of $m_{\widetilde{t}_{1}}$ [14, 15]. To compare behavior of the stop production with the SM processes, curves obtained by the $\lambda_{131}^{\prime}=0.1$ are added to both $e^{-}$and $e^{+}$cases. Dependence of the cross section on the polarization is stronger in the stop production than in the SM processes. For example, cross sections of the stop production with fully polarized $e_{L}^{-}$and $e_{R}^{+}$beams are two times as much as unpolarized ones while those of the SM increase by only $\sim 30 \%$. One can see from this figure that righted-handed $e^{+}$beams are efficient to produce the stop in our model.

In order to study production processes of the stop through the s- and the u-channel ( Fig.1), we calculate separately these contribution for unpolarized beams. Fig.3 shows cross sections of the stop production of the s- and the $\mathrm{u}$-channel processes together with the SM background as a function of the mass of the stop $m_{\widetilde{t_{1}}}$. Here we assume that $\lambda_{131}^{\prime}=0.01$, 0.03 and other conditions are the same as Fig.2. If the mass of the stop is larger than the center-of-mass energy of the $e p$ system, $\sqrt{s}$, a peak in $x$ distribution disappears. However contribution of the stop still remains in the whole region of $x$ distributions due to tails of the $\mathrm{s}$-channel resonance and the $\mathrm{u}$-channel process. From Fig.3 we can see that contribution of the stop production from the $\mathrm{u}$-channel process is of the order of a few $\mathrm{fb}$. It is larger for $e^{-}$beams than for $e^{+}$beams. This can also be understood from the structure of the $\mathrm{RB}$ interaction. While the $e^{-}$collides with valence $d$-quarks in the proton, the $e^{+}$collides only with sea $\bar{d}$-quarks in u-channel diagram. On the other hand contribution of the stop from the tails of the s-channel resonance is still larger than the u-channel contribution in both $e^{-}$and $e^{+}$cases. This is due to the broad distribution for the large stop mass.

Fig. 4 shows an example of $x$ distributions of scattered $e^{+}$for fully polarized rightedhanded beams and unpolarized beams under the condition of the integrated luminosity of $1 \mathrm{fb}^{-1}$, where $m_{\widetilde{t}_{1}}=200 \mathrm{GeV}, \lambda_{131}^{\prime}=0.015$ are assumed as typical values for our interest. Peaks at $x=0.41$ due to the stop over the smooth behavior of the SM events are more enhanced in the case of polarized $e^{+}$beams than unpolarized ones. This implies a possibility to search for the stop at the level of $\lambda_{131}^{\prime}=0.015$ for $e^{+}$beams.

The reach in $\lambda_{131}^{\prime}$ for the wide range of masses of the stop $160-400 \mathrm{GeV}$ is studied with an integrated luminosity of $1 \mathrm{fb}^{-1}$. Lower limits on $95 \%$ confidence-level exclusion for $\lambda_{131}^{\prime}$ versus the mass of the stop is shown in Fig.5 for fully polarized $e_{R}^{+}$beams and unpolarized ones. We have imposed here the condition of $S / \sqrt{B}=1.96$ where $S$ and $B$ denote the number of stop events and that of the SM background events respectively. Excluded region $m_{\widetilde{t}_{1}} \leq 240 \mathrm{GeV}$ given by the Tevatron experiments is also shown in Fig.5 
[16]. We see from this figure that we can search for the stop having the mass in the range of 240-280 GeV at the level of $\lambda_{131}^{\prime}>0.01-0.05$ with fully polarized $e_{R}^{+}$beams. The upgraded HERA would meet our expectation. We can set the $95 \%$ confidence-level exclusion limit in this parameter region if no signal of the stop is observed.

It is important to identify the stop above the backgrounds due to the SM process by using kinematical variables characteristics to the stop resonance. Differential cross sections of the s-channel stop production are not sensitive to $Q^{2}$ in contrast to the strong decrease for the SM when $Q^{2}$ increases. It is possible to estimate the contribution of the stop production by using $y\left(=Q^{2} / s x\right)$ distributions of $e^{+}$for $e^{+} p \rightarrow e^{+} X$. Fig.6 shows $y$ distributions of the $e^{+}$at the fixed $x=x_{\text {peak }}$ for the stop production together with the SM. Here we assume $\lambda_{131}^{\prime}=0.04$ and $m_{\widetilde{t}_{1}}=250 \mathrm{GeV}$, which corresponds to $x_{\text {peak }}=0.634$. We see from this figure that possible events in the $y$ distribution at large $y$ could be the signature of the stop production.

\section{Concluding remarks}

We have investigated the production rate of the stop with polarized electron and positron beams at HERA in the framework of the MSSM with RB interactions. Fully polarized $e_{L}^{-}$or $e_{R}^{+}$produces the stop two times as much as unpolarized beams, while they increase the SM backgrounds by $\sim 30 \%$ in comparison with unpolarized ones. The $e^{+}$beams are more efficient to produce the stop than $e^{-}$beams because of the coupling of $e^{+} d$ and $e^{-} \bar{d}$ for the stop in the model. We can search for the stop using $e_{R}^{+}$beams for masses in the range of $240-280 \mathrm{GeV}$ at the level of $\lambda_{131}^{\prime}>0.01-0.05$. Experimentally, these parameter ranges have not been covered yet. If no signal of the stop is observed, we can set $95 \%$ confidence-level exclusion limit in this parameter region. We see that the high $y$ region is effective to identify the stop signal. The present limits would be influenced by the limited degree of polarization and detection efficiency.

It is pointed out by T.Plehn et al. [17] that the contribution of next-to-leading order (NLO) QCD corrections are important in the cross sections for the formation of scalar resonances, leptoquarks or squarks in $e^{ \pm} p$ collisions at HERA. They showed that the $K$-factors increase the production cross sections by up to $30 \%$ depending mildly on the mass of the resonances, if the target quarks are valence quarks. In our present study, the sea $(\bar{d})$ quark scattering and the off-resonance ( $\mathrm{s}-$ and $\mathrm{u}$-channels) contributions play an important role. It is needed to estimate the NLO corrections to the whole scattering processes $e^{ \pm} p \rightarrow e^{ \pm} X$.

\section{Acknowledgements}

This work was supported in part by the Grant-in-Aid for Scientific Research from the Ministry of Education, Science and Culture of Japan, No. 10440080. 


\section{References}

[1] "Supersymmetry, Supergravity And Particle Physics", H.P.Nilles, Phys. Rep. 110, 1 (1984).

[2] "The Search For Supersymmetry: Probing Physics Beyond The Standard Model", H.E.Haber and G.L.Kane, Phys. Rep. 117, 75 (1985).

[3] K. Hikasa and M. Kobayashi, Phys. Rev. D36, 724 (1987).

[4] C. Adloff et al., H1 Collab., DESY preprint, DESY 97-24, hep-ph/9702012.

[5] J. Breitweg et al., ZEUS Collab., DESY preprint, DESY 97-25, hep-ph/9702015.

[6] V. Barger, G. F. Giudice and T. Han, Phys. Rev. D40, 2987 (1989) ; W. Buchmüller, R.Ruckl and D.Wyler, Phys. Lett. B191, 442 (1987)

[7] "Parton and forces: high $Q^{2}$ DIS at HERA" K.Long, talk presented at the workshop on "Electron Proton Interaction with High Transverse Energy" 27/28 March,2000 (KEK).

[8] J. Ellis and S. Rudaz, Phys. Lett. 128B, 248 (1983); G. Altarelli and R. Rückl, Phys. Lett. 144B, 126 (1984); I. Bigi and S. Rudaz, Phys. Lett. 153B, 335 (1985).

[9] F. Abe et al. (CDF Collab.), Phys. Rev. Lett. 74, 2626 (1995) ; S. Abachi et al. (D0 Collab.), Phys. Rev. Lett. 74, 2632 (1995).

[10] "Stop Resonances at HERA in R-parity Breaking Models", T. Kon and T. Kobayashi, Phys. Lett. B270, 81 (1991).

[11] T. Kon, T. Kobayashi, S. Kitamura, K. Nakamura and S. Adachi, Z. Phys. C61, 239 (1994) ; T. Kobayashi, S. Kitamura and T. Kon, Int. J. Mod. Phys. 1111875 (1996).

[12] H.L.Lai et al., Phys. Rev. D51, 4763 (1995).

[13] S.Kawabata, Comput. Phys. Commun. 41, 127 (1986).

[14] "High $Q^{2}$ Physics at HERA and Searches for New Particles", T. Matsushita, E.Perez, R. Rückl, Preprint WUE-ITP-98-056(1998), Contribution to the 3rd UK Phenomenology Workshop on HERA Physics, St. John's College, Durham, UK, September 1998.

[15] S. Davidson, D. Bailey and B. A. Campbell, Z. Phys. C61, 613 (1994) ; P Langacker, Phys. Lett. B256, 277 (1991)

[16] Leptoquark Limit Combination Working Group (for the CDF and D0 Collaborations), hep-ex/9810015

[17] T.Plehn, H.Spiesberger, M.Spira and P.M.Zerwas, Z. Phys. C74, 611 (1997) 


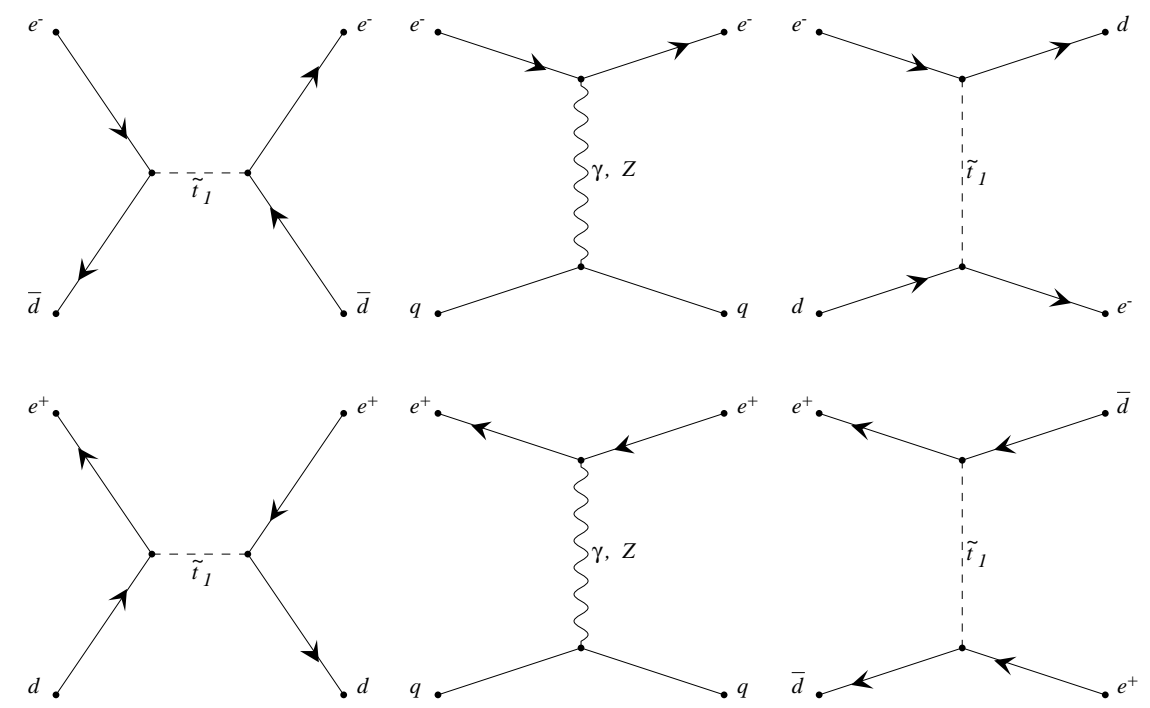

Figure 1: Feynman diagrams for sub-processes $e^{ \pm} q \rightarrow e^{ \pm} q$. 

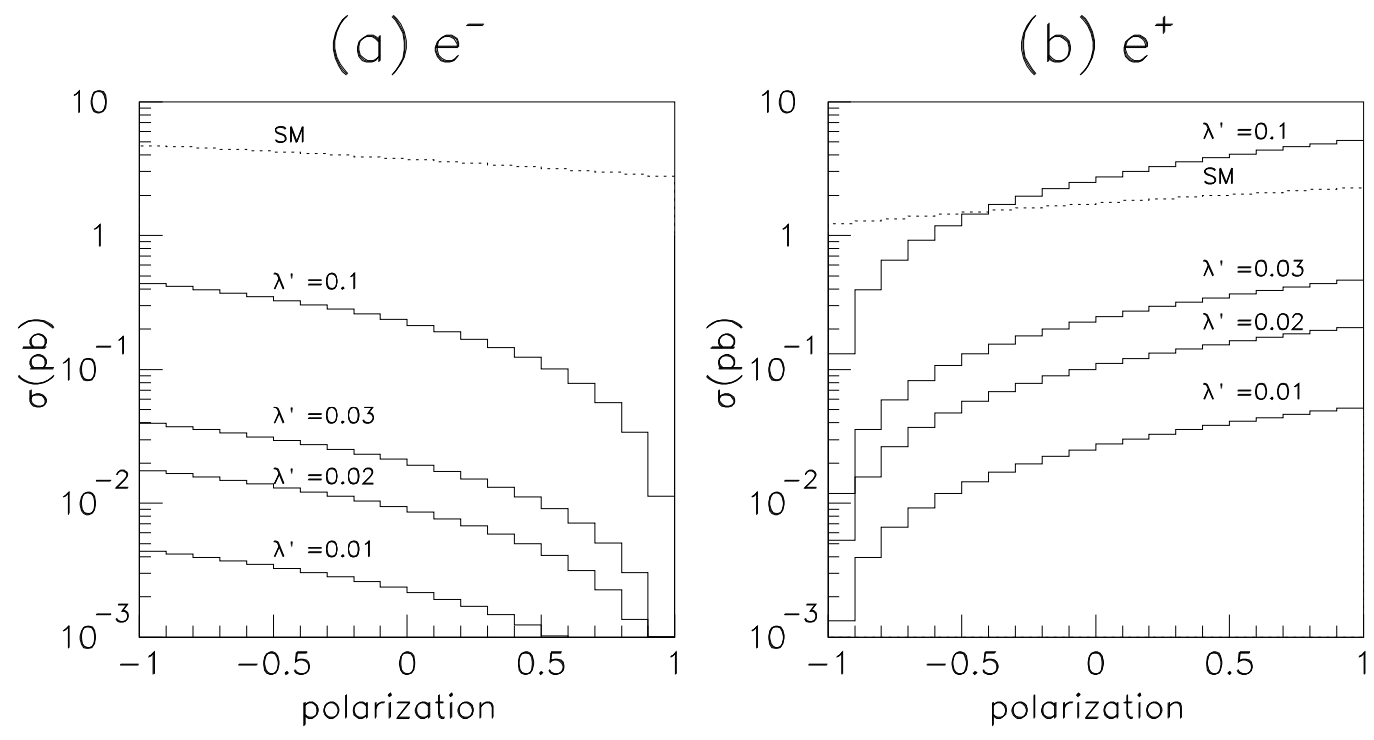

Figure 2: Cross sections of the stop production and the SM process of (a) $e^{-}$and (b) $e^{+}$beams as a function of polarization in $e p$ collisions. Polarization of $-1,0,1$ corresponds to fully polarized left-handed beam, unpolarized beam, and fully polarized right-handed beam respectively. Solid lines and dotted lines show the cross sections of stop production and that of the SM process, respectively. $Q^{2}>10^{4}(\mathrm{GeV} / \mathrm{c})^{2}, m_{\widetilde{t}_{1}}=200 \mathrm{GeV}, \lambda_{131}^{\prime}=$ $0.01,0.02,0.03,0.1$ and $\theta_{t}=0.0$ are assumed. 

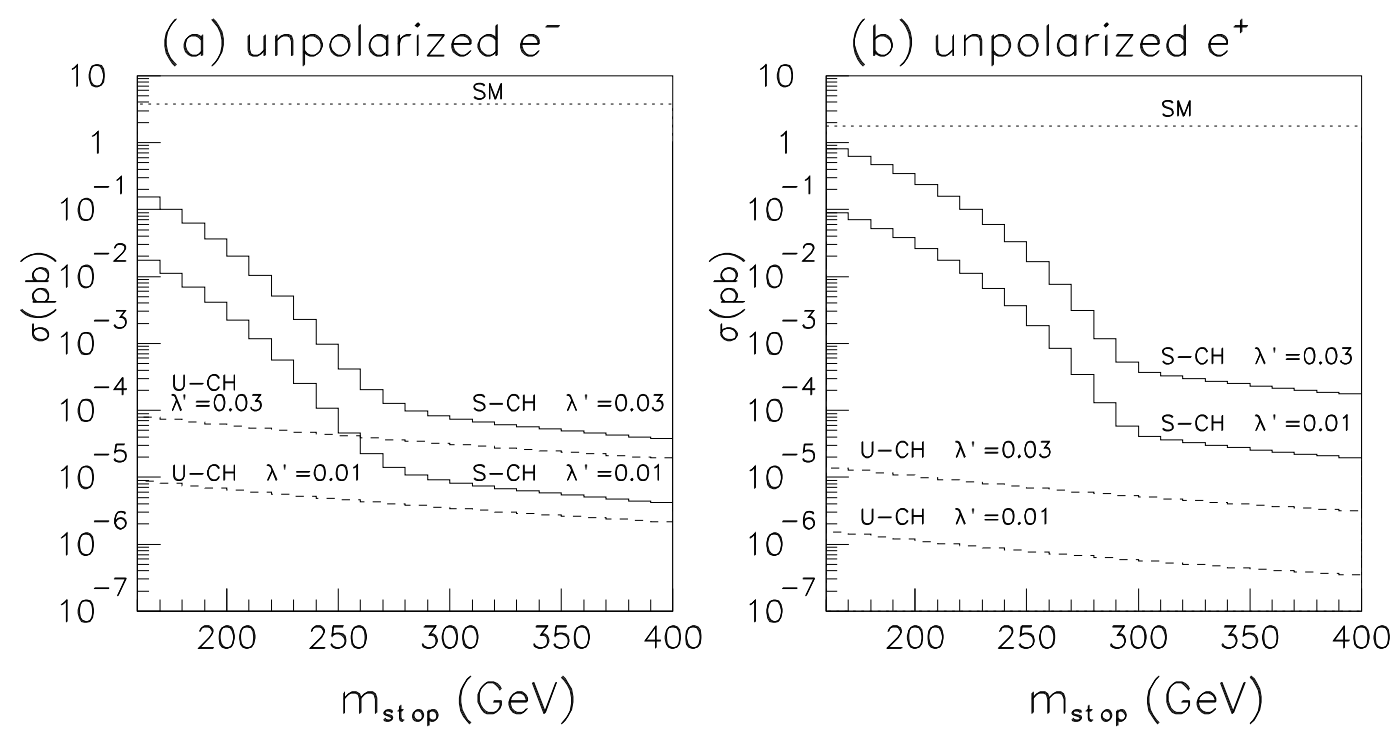

Figure 3: Cross sections of the stop production due to s-channel process (solid lines), u-channel process (dashed lines) and the SM background (dotted lines) as a function of the mass of the stop $m_{\widetilde{t}_{1}}$ for (a) $e^{-}$unpolarized beams and (b) $e^{+}$unpolarized beams. $Q^{2}>10^{4}(\mathrm{GeV} / \mathrm{c})^{2}, \lambda_{131}^{\prime}=0.01,0.03$ and $\theta_{t}=0.0$ are assumed in both cases. 
(a) unpolarized $e^{+}$

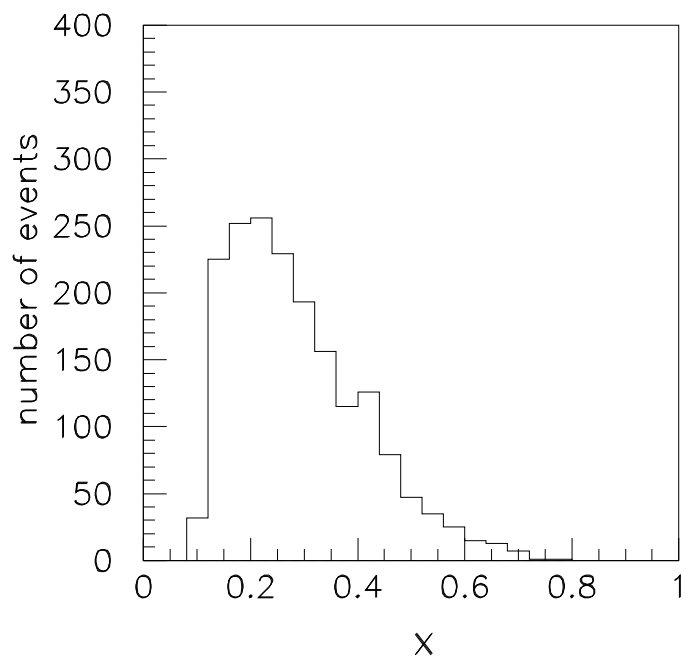

(b) polarized $\mathrm{e}_{\mathrm{R}}{ }_{\mathrm{R}}$

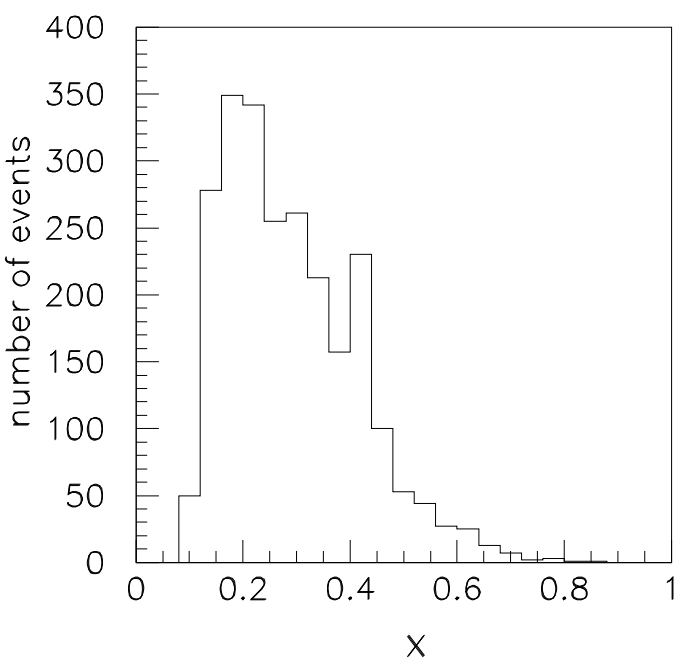

Figure 4: $x$ distributions of $e^{+}$of the processes $e^{+} p \rightarrow e^{+} X$ with (a) unpolarized $e^{+}$ beams and (b) fully polarized $e_{R}^{+}$beams. $Q^{2}>10^{4}(\mathrm{GeV} / \mathrm{c})^{2}, m_{\widetilde{t}_{1}}=200 \mathrm{GeV}, \lambda_{131}^{\prime}=$ 0.015 and $\theta_{t}=0.0$ are assumed with $\mathcal{L}=1 \mathrm{fb}^{-1}$. 


\section{5\% CL exclusion limit}

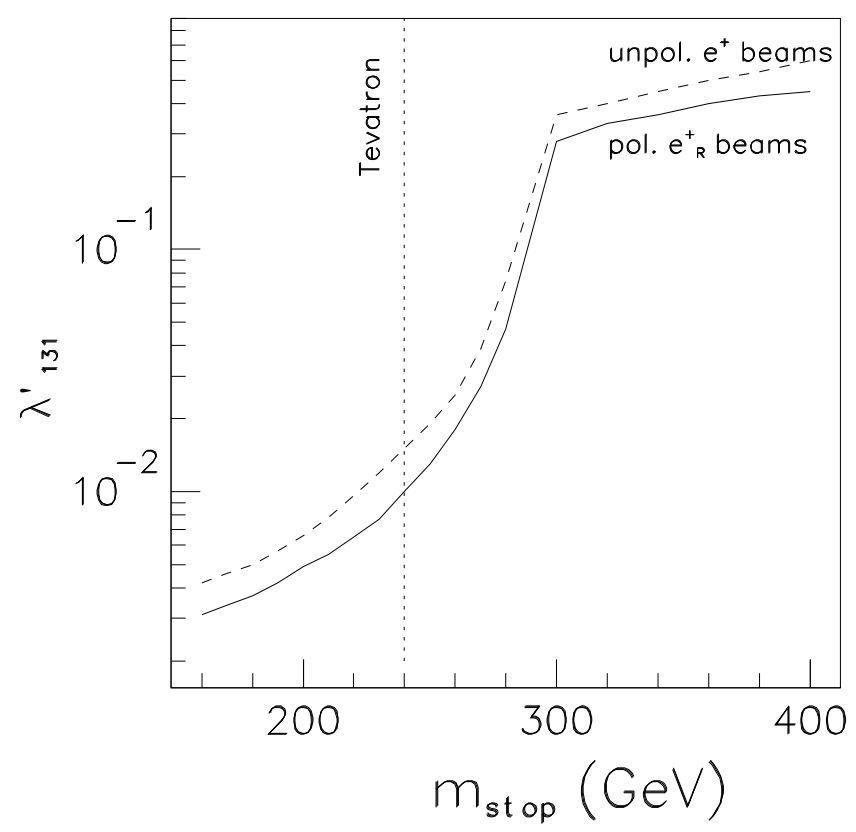

Figure 5: $95 \%$ confidence-level exclusion limit $(S / \sqrt{B}=1.96)$ for $\lambda_{131}^{\prime}$ versus stop mass $m_{\widetilde{t}_{1}}$ in run of HERA with an integrated luminosity of $1 \mathrm{fb}^{-1}$ for fully polarized $e_{R}^{+}$beams (solid line) and unpolarized $e^{+}$beams(dashed line). Upper region of each curve is excluded. A dotted vertical line is a lower bound of $m_{\widetilde{t}_{1}}$ of Tevatron 16. 

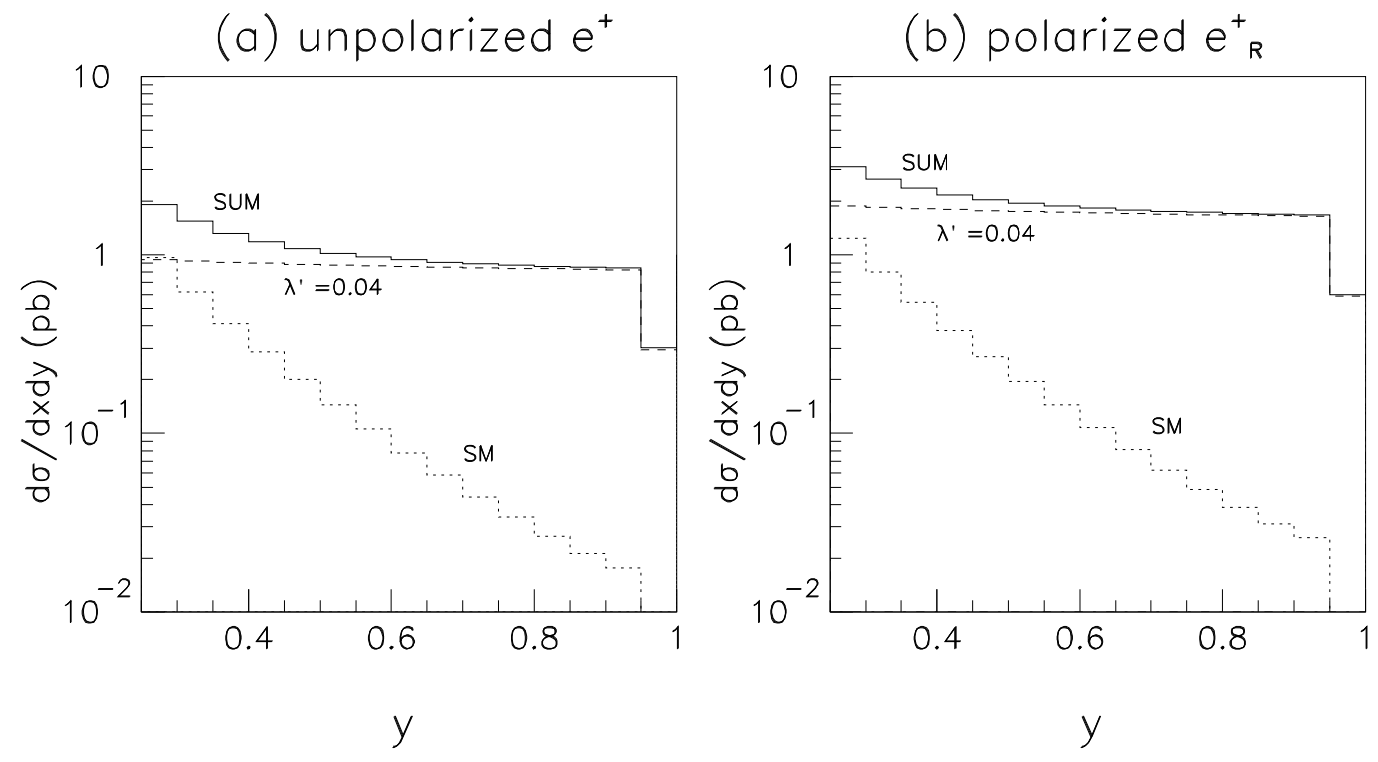

Figure 6: $y$-distributions of $e^{+}$of the process $e^{+} p \rightarrow e^{+} X$ for (a) unpolarized $e^{+}$beam and (b) fully polarized $e_{R}^{+}$beams, where $x$ is within the interval $0.634 \pm 0.02$ which corresponds to $m_{\widetilde{t}}=250 \pm 4 \mathrm{GeV} . Q^{2}>10^{4}(\mathrm{GeV} / \mathrm{c})^{2}, \lambda_{131}^{\prime}=0.04$ and $\theta_{t}=0.0$ are assumed. Dashed, dotted and solid lines show the distributions for the process of $\widetilde{t}_{1}$ production, the SM process and sum of them, respectively. 